\title{
3D Laplacian Surface Deformation for Template Fitting on Craniofacial Reconstruction
}

\author{
Putu Hendra Suputra \\ Department of Electrical Engineering \\ Institut Teknologi Sepuluh Nopember, \\ Surabaya, Indonesia; \\ Department of Informatics \\ Universitas Pendidikan Ganesha, \\ Singaraja, Indonesia \\ hendra.suputra@undiksha.ac.id
}

\author{
Anggraini Dwi Sensusiati \\ Department of Radiology \\ Universitas Airlangga, Surabaya, \\ Indonesia \\ anggraini-d-s@fk.unair.ac.id
}

\author{
Eko Mulyanto Yuniarno \\ Department of Electrical Engineering \\ Department of Computer Engineering \\ Institut Teknologi Sepuluh Nopember, \\ Surabaya, Indonesia \\ ekomulyanto@ee.its.ac.id
}

\author{
Mauridhi Hery Purnomo \\ Department of Electrical Engineering \\ Department of Computer Engineering \\ Institut Teknologi Sepuluh Nopember, Surabaya, \\ Indonesia \\ hery@ee.its.ac.id
}

\author{
I Ketut Eddy Purnama \\ Department of Electrical Engineering \\ Department of Computer Engineering \\ Institut Teknologi Sepuluh Nopember, Surabaya, \\ Indonesia \\ ketut@te.its.ac.id
}

\begin{abstract}
Computer-based craniofacial reconstruction utilizes medical imaging for forensic identification and medical reconstruction surgery, enables sophisticated three-dimension object representation and visualization. Reconstruction process involves deformation in their imagery how the shape of a person's face based on its properties and landmark correspondences. We propose the Laplacian surface deformation to deform the facial template to the cranial surface on several constraints that simulates fitting face to the skull using landmark correspondences. The range of variation of the landmarks on the human face lies in narrow intervals. In other words, small differences can affect the shape and facial expressions. Thus, a Laplacian surface model gives better result than a volumetric Laplacian in the matter of accuracy.
\end{abstract}

\section{CCS Concepts}

- Computing methodologies $\rightarrow$ Shape representations.

\section{Keywords}

Laplacian; surface-deformation; volumetric-deformation; craniofacial reconstruction; biomedical; surface editing.

\section{INTRODUCTION}

Craniofacial reconstruction is a process that demands time and expertise of the experts (surgeons, artists, and morphological anatomists). This process often consumes time and requires high

Permission to make digital or hard copies of all or part of this work for personal or classroom use is granted without fee provided that copies are not made or distributed for profit or commercial advantage and that copies bear this notice and the full citation on the first page. Copyrights for components of this work owned by others than ACM must be honored. Abstracting with credit is permitted. To copy otherwise, or republish, to post on servers or to redistribute to lists, requires prior specific permission and/or a fee. Request permissions from Permissions@acm.org.

ICCCM'20, July 17-19, 2020, Singapore, Singapore

(C) 2020 Association for Computing Machinery.

ACM ISBN 978-1-4503-8766-8/20/07 ...\$15.00

https://doi.org/10.1145/3411174.3411175 precision. The expected results are morphological estimates that are close to the actual shape [1]. The purpose of craniofacial reconstruction is to recreate what someone looks like based on one's remnants (skull).

Manual reconstruction method requires anatomical and artistic expertise so that it is subjective and highly dependent on the expert. Interpretation results from two experts or artists produce different faces where the differences widely vary [4]. Furthermore, the sequential manual method is time-consuming and challenging to process in parallel.

Computer-aided techniques for face reconstruction have a general model workflow; it is the process inspired by how conventional (manuals) approaches are carried out: (a) Firstly, the skull remnant of a person is analyzed by experts to determine the attributes of the specimen such as gender, age, race, or ethnicity; (b) these attributes are then linked to digital copies of skulls obtained through CT image scans or other modalities. Then (c) a craniofacial model (CFM) is made whose format can differ from one technique to another, emphasizing the technique used. The CFM must have all the potential needed by the technique, and able to provide information about the skull so that information can be extracted. CFM represents the skull in the analysis and reconstruction process, as experts use original skulls. Defining CFM is the most crucial stage in craniofacial reconstruction. In this stage, a model is built, in which the model must able to encode information and knowledge about the human skull and the shape of the overlying face related to it. Then (d) Identification of craniofacial relation on skull representation is the next step to (e) obtain properties needed for the reconstruction process. The last component is texturing and rendering (f) to add visual presentation for the final result.

The reconstruction process involves the process of deformation in their imagery how the shape of a person's face based on properties is obtained from the previous stages. Some techniques can be used to perform reconstruction for identification, such as shape matching, landmark-matching (Skull-Face Overlay), and mesh deformation $[1,3,8,9,12,13]$. However, the deformation process 
takes an important role in defining the shape of the result. Volumetric Laplacian deformation was used in [9] but only partially on the nose region for cosmetic surgery reconstruction. The work on [3] although empowering template deformation, is an automatic shape-matching process that does not require any landmark correspondences. Face template deforming was also implemented in $[8,12]$, their method applied Hermite Radial Basis Function (HRBF) and moving least squares (MLS) deformation, respectively.

As of now, there has been no paper reporting about deformation for craniofacial reconstruction that deforms the face template to the skull using the Laplacian model, based on landmark correspondences. In this paper, we propose the Laplacian surface deformation to deform craniofacial template on several constraints that simulates a-priori landmark correspondences.

The range of variation of the landmarks on the human face lies on narrow intervals. In other words, small differences can affect the shape and facial expressions. Accuracy measured by comparing the distance between user constrain and its corresponding final position as an absolute error $\left(\left|v_{i}^{\prime}-u_{i}\right|\right)$. Thus, a Laplacian surface model applied as it performs better than a Laplacian volumetric model in term of accuracy.

\section{PROPOSED SYSTEM}

Surface processing and representation on geometric fields, especially in computer-related works is one of the primary and standard graphical procedures. A strong representation gives a better confidence to proceed to the next stage. In this work, a Laplacian model is used as a surface representation to mimic an elastic surface on human face reconstruction case.

\subsection{Data Preparation}

Computer tomography (CT) is considered to provide good results because it has advantages over MRI for bone-related works. It is easier to obtain, producing consistent image quality, giving better bone detail, especially concerning distinguishing soft tissue with bone [15]. Point clouds are extracted from the CT image on facial region area, since that area contains most biometrical information of human face [6]. Down sampling and noise removal is one essential step in 3D data processing [11]. The number of calculations that appear will increase along with the number of vertices on the model. To be able to run the computation into acceptable time, it is to be taken into consideration, trading off surface detail for performance at an acceptable level.

CT images of the head are processed to extract the outer surface of the face. The outer contour extraction delivers point clouds, which consist of 400 thousand points on average. Laplacian deformation requires every point's neighborhood information in the form of adjacency matrix. To be able to process point clouds well on computer machines today, considering the nature of Laplacian model calculations that require large matrices in proportion to the number of vertices, it is necessary to down sample the point clouds. Down sampling the coordinate sequence (by an integer factor) is a convenient way to minimize computing loads (Figure 1)

Extraction of the outer surface of the human skull was conducted in [19] which managed to extract the skull region as point clouds on DICOM formatted CT images. The outer-contour extraction method was proposed, which extracted parts of the head on every CT slice that can be seen from four views, scanning for the outer contour starting from left, right, top and bottom side. The sequential process of extracting outer contour as point clouds was conducted. Prior to, a region of interest (RoI) is defined by a mask, since the shape and directional of the patient's head are uniform. Point clouds are represented as a triangulated graph. It is constructed by triangulated point network as an undirected graph $G=(V, E)$, where $V=\left(v_{1}, v_{2}, \cdots, v_{n}\right)$, set of vertices connected each other with a set of edge $E$.

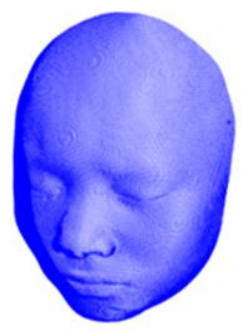

(a)

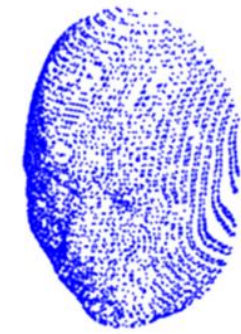

(b)

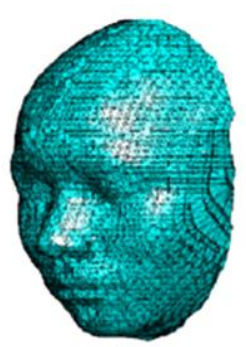

(c)
Figure 1. Surface acquisition and representation: (a)

Point clouds acquired from multi-slice CT image; (b)

Decreased sample rate point clouds on a ratio that keeps the essential detail of human face; (c) Triangulated surface of resampled point clouds.

\subsection{Laplacian Model}

Laplacian surface editing is detail-preserving techniques based on manipulation of differential coordinates and solves a system to obtain the deformed surface. This natural and intuitive characteristic is suitable for simulating elastic surface deformation. In this work, we research and implement the Laplacian coordinates on the deformation of a human face surface. Firstly, point clouds are extracted from the CT image as a set of three dimension Cartesian coordinates [10,19]. Surface triangulation is the next important step as the basis for finding neighborhood information, and also for rendering the deformed surface $[5,7,14]$. Thus, we can use first differential coordinates and several fixed constraints to solve deformed coordinates. Finally, simulation is presented by rendering the surface and determining its qualitative measurement.

Facial surface deformation proposed in this work has a basis on the characteristic of elastic surface (detail-preserving surface editing) [2, 17]. Therefore, it needs a model that reckons neighborship connection. The Laplacian matrix is applied in this proposal as it can represent neighborhood connection. This representation has the capability of describing and defining the local differential shape for geometry representation [18].

Laplacian matrix of an undirected non-loop graph $G$ is a symetric

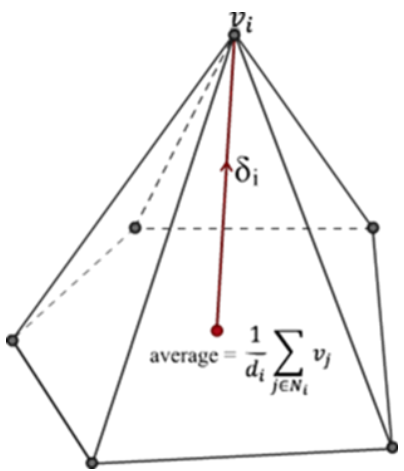

Figure 2. The $\delta$-coordinate of $v_{i}$. 
matrix. In performing Laplacian surface deformation, the first differential ( $\delta$-coordinates of the $i$-th vertex, $v_{i}$ ) must be constructed from the Cartesian coordinates (Figure 2) which models the elastic characteristic of this method. The $\delta$-coordinate is denoted as the differences between the absolute coordinate of $v_{i}$ and the center of mass of its immediate neighbors in the mesh [16].

$$
\begin{aligned}
\delta_{i}=\left(\delta_{i}^{(x)}, \delta_{i}^{(y)}, \delta_{i}^{(z)}\right) & =v_{i}-\frac{1}{|N(i)|} \sum_{j \in N_{i}} v_{j} \\
& =v_{i}-\frac{1}{d_{i}} \sum_{j \in N_{i}} v_{j}
\end{aligned}
$$

where, $N(i)=\{j \mid(i, j) \in E\},|N(i)|=$ number of immediate neighbors of $v_{i}$, which can also be notated as $d_{i}$ (degree of $v_{i}$ ). Let $D$ be the diagonal matrix such that $D_{i i}=d_{i}$ or $D=$ $\operatorname{diag}\left(d_{1}, d_{2}, \cdots, d_{n}\right)$ is a diagonal matrix which formed by the degrees of each vertex, and $A$ is an adjacency matrix which shows connection value of every vertex to each other. Then the matrix transforming the absolute coordinates to relative coordinates is denoted by

$$
L=I-D^{-1} A
$$

\subsection{Laplacian Deformation Algorithm}

The Laplacian deformation is carried out with process which including the making of Laplacian coordinates and adding fixed constraints onto the Laplacian coordinate and solving back the remaining coordinates as a result of this addition. Constructing a differential representation is the first step before proceeding to the deformation step. The Laplacian coordinate is defined as a differential coordinate which every vertex in the mesh has a relationship as the difference or distance between the position of the vertex and the average-vertex of the neighbors. Laplacian coordinate $\delta_{i}, \delta$-coordinates of $v_{i}$, is defined by deformation gradients as

$$
\delta_{i}=\mathcal{L}\left(v_{i}\right)
$$

is the difference from the average of neighbors for a single vertex from equation (1), where $N_{i}$ : adjacent vertices, and $D_{i}$ : the degree of a vertex. By the equation (1) and (2), we have equation (4).

$$
\mathcal{L}\left(v_{i}\right)=v_{i}-\frac{1}{d_{i}} \sum_{j \in N_{i}} v_{j}
$$

The difference from the average of neighbors for a single vertex is also referred as one-ring neighborhood. Laplacian coordinate computation requires a matrix that contains the one-ring neighborhood information for all vertex, and calculates a one-ring neighborhood matrix formulation. An adjacency matrix is needed as a reference for neighboring connection of the vertex. Detailpreserving surface means that it takes into account neighbor relationships during the deformation process. Laplacian coordinate $\delta_{i}$ relies on the information of the adjacency matrix $A$. The adjacency matrix $A$ for each point consists of the binary value [1,0], a value 1 if $v_{i}$ and $v_{j}$ are connected, and a value 0 for vice versa. The adjacency matrix for the graph in Figure 3 denoted below. Matrix $A$ is a symmetrical matrix that each row or column represents the connection of one vertex to another,

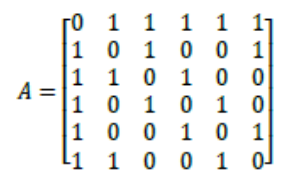

From the adjacency matrix, the degree of each vertex can be formulated into a diagonal degree matrix for computational purposes. The degree matrix can be denoted as a diagonal matrix $=\operatorname{diag}\left(d_{1}, d_{2}, \cdots, d_{n}\right)$ where $d_{i}$ is a sum of column- $i$ of the adjacency matrix. For computational purpose, we need to transform the Laplacian coordinates in a matrix form. Let vertices matrix $V=\left(v_{1}, v_{2}, \cdots, v_{n}\right)^{T}$, differential matrix as $\Delta=$ $\left(\delta_{1}, \delta_{2}, \cdots, \delta_{n}\right)^{T}$ and recall the equation (3) and equation (2) then the differential matrix (for all vertices) can be notated as follows

$$
\begin{aligned}
\Delta & =L V \\
\Delta & =\left(1-D^{-1} A\right) V
\end{aligned}
$$

The surface editing process is conducted by adding a user constraints to the Laplacian coordinates, also adding a revised value to the corresponding differential matrix, which are the position-adjustment of the user constraints. By modifying the Laplacian coordinates and its corresponding user-specified vertices (as fixed user-constraints), we look for how the changes occur to the vertex position $\left(V^{\prime}\right)$. Vertex $V^{\prime}$ position is obtained by multiplying the Laplacian coordinate matrix with the differential matrix. Solving remaining vertices $V^{\prime}$ from the equation (5):

$$
\begin{aligned}
L V & =\Delta \\
\Delta & =\left(1-D^{-1} A\right) V
\end{aligned}
$$

Pseudoinverse $L^{+}=\left(L^{T} L\right)^{-1} L^{T}$ is used since $L$ cannot be inverted after the addition of the user-constraints. Operational computation for solving $V^{\prime}$ becomes:

$$
V^{\prime}=\left(L^{T} L\right)^{-1} L^{T} \Delta
$$

This deformation maintains the integrity of shape and deforms it to minimize errors, which equivalent to optimization goal minimizing residual least square error $[16,18]$ :

$$
E\left(V^{\prime}\right)=\underset{v^{\prime}}{\operatorname{argmin}} \sum_{i=1}^{n}\left\|\delta-\mathcal{L}\left(v_{i}^{\prime}\right)\right\|^{2}+\sum_{i=m}^{n}\left\|v_{i}^{\prime}-u_{i}\right\|^{2}
$$

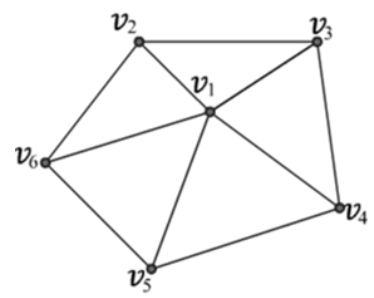

Figure 3. Connected graph of the $\delta$-coordinate (Figure 2).

\section{MAIN RESULTS}

The editing process includes stationary anchors and some chosen handles, which act as an added constraint manipulated by the user. The deformation is applied to the face point clouds. The deformation is sought as natural as possible, mimicking the characteristics of human biometric elasticity. The deformation is performed by adding the fixed constraints (as points or blocks). The fixed constraints can be a stationary anchor or a user handle. Stationary anchors are the points in which the deformation process is strived to remain in their original position. While the handles are the key points planned to be altered. Both stationary anchors and handles have the same role, as a designated target points, which comprehensively affect all other points according to their connectivity to each other. The stationary anchors maintain their position and other points position around it, to stay in their original place, while the handle will draw the surrounding points. 
Fitting process, the position adjustment due to tensile force between points, is approximated by equation (5) and (6). Final coordinates are the optimal result of the fixed constraints adjustment. So, there is a difference $\left(v_{i}^{\prime}-u_{i}\right)$ between the designated position (user fixed constraints $u_{i}$ ) and the final position $\left(v_{i}{ }^{\prime}\right)$ as shown in Figure 4.

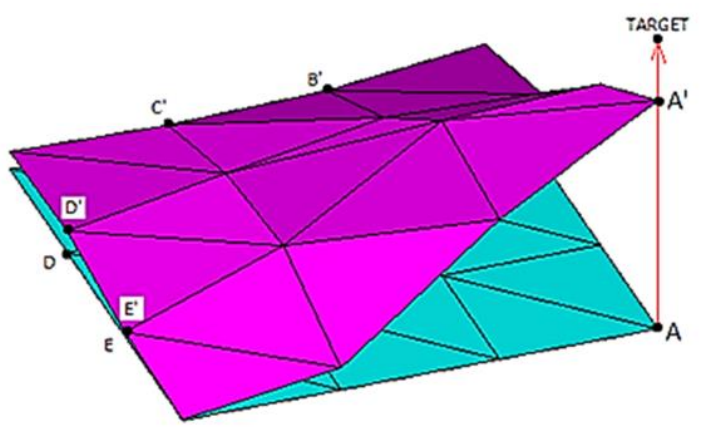

Figure 4. Mesh reconstruction).

The Laplacian surface deformation for facial reconstruction can be implemented using two models, surface and volumetric approaches. Both use similar concepts of adjacency relations but differ in the nature of neighborliness. Neighboring on the Laplacian surface is built on the principle of triangulation of the outermost surface points. The triangulation builds a relationship between planar field of three points, along with another field of triangulation to form a surface. Unlike the surface model, the volumetric model forms connectivity between four points with tetrahedron (the smallest unit of volume formed on four points). What is the difference between the two models if you apply Laplacian deformation? As mentioned earlier, the Laplacian deformation is based on the neighbor relationship (equation 2). The volumetric model naturally contributes more neighbor values compared to the surface model; if looking back at the equation, it surely makes a difference.

Experiments are carried out on both models on toy example. A closed dome shape (Figure 5) with 1562 points, is chosen because it is closest to the basic shape of the face template that will be used. The surface model performs better in term of accuracy, considering that the depth variations of landmarks on human faces are at narrow intervals, where small differences will result in different facial impressions.

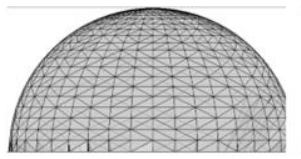

A

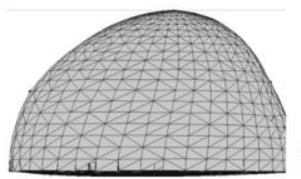

C

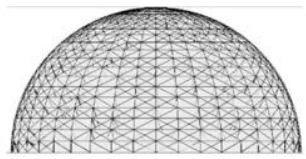

B

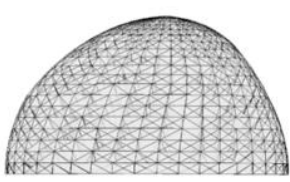

$\mathrm{D}$
Figure 5. Dome shape with 1562 points for surface (A) and volumetric (B) Laplacian performance comparison; deformed dome for both surface and volumetric $(\mathrm{C}$ and D).

The experimental scenario is designed with five fixed user constraints provided that the distances between constraints in each mode (A, B, C, D) are enlarged (getting away from each other) in order to determine the effect of the involvement of points between constraints on the accuracy produced $\left(v_{i}^{\prime}-u_{i}\right)$. Seven fixed constrain also applied to give confidence in using more fixed constraints to the accuracy.

Editing is done by making small changes for each constraint simulating changes that occur in the process of fitting face templates based on corresponding face landmarks. Variations in position and depth of landmarks move in insignificant ranges. The experiments number 1 and 2 use random coordinate shifts in small ranges \pm 10 for each axis. Larger ranges \pm 20 are used for the experiments number 3 and 4 . While number 5 is only one constraint that is shifted as far as 20 points on the z-axis, and experiment 6 and 7 are using seven fixed constraints. In mode A, the distance between constraints is arranged very close (minimal), while in mode $\mathrm{B}$ to $\mathrm{D}$, the distances of the constraint are arranged farther away to each other on the mesh, that means the farther the distance, the more points are involved in the connection. Both the surface and volumetric models perform smaller mean absolute error when the constraints placed away, while the use of five or seven constraints does not have a significant influence on this experiment. The farther the distance between constraints, the more the points connected between them should be. Of course, there will be an adjustment in the number of neighboring relations involved in between. Even though the constraints position is the same in both models, it seems that the difference in the number of neighbor relations affects the difference in performance produced.

Table 1. Performance comparison in term of accuracy on surface and volumetric model

\begin{tabular}{|c|c|c|c|c|c|c|c|c|c|c|c|c|}
\hline \multirow{3}{*}{ No. } & \multicolumn{12}{|c|}{ Mean absolute error } \\
\hline & \multicolumn{3}{|c|}{$\mathbf{A}$} & \multicolumn{3}{|c|}{$\mathrm{B}$} & \multicolumn{3}{|c|}{$\mathbf{C}$} & \multicolumn{3}{|c|}{$\mathrm{D}$} \\
\hline & $\mathbf{S a}$ & Va & Sa:Va & $\mathrm{Sb}$ & $\mathrm{Vb}$ & $\mathrm{Sb}: \mathrm{Vb}$ & Sc & Vc & Sc:Vc & $\mathrm{Sd}$ & $\mathrm{Vd}$ & Sd:Vd (\%) \\
\hline 1 & 1.24 & 1.68 & 26.19 & 0.32 & 0.66 & 51.03 & 0.28 & 0.51 & 44.62 & 0.24 & 0.41 & 40.90 \\
\hline 2 & 0.87 & 1.10 & 20.30 & 0.21 & 0.42 & 51.14 & 0.12 & 0.22 & 44.34 & 0.10 & 0.15 & 33.51 \\
\hline 3 & 2.13 & 3.35 & 36.26 & 0.83 & 1.75 & 52.56 & 0.63 & 1.13 & 44.25 & 0.52 & 0.86 & 39.48 \\
\hline 4 & 2.86 & 3.65 & 21.56 & 0.81 & 1.57 & 48.26 & 0.45 & 0.75 & 40.03 & 0.32 & 0.50 & 34.77 \\
\hline 5 & 1.76 & 2.43 & 27.63 & 0.48 & 1.21 & 59.87 & 0.09 & 0.29 & 68.55 & 0.02 & 0.08 & 70.84 \\
\hline 6 & 1.57 & 1.99 & 20.98 & 0.50 & 0.92 & 45.55 & 0.46 & 0.76 & 40.25 & 0.34 & 0.50 & 31.82 \\
\hline 7 & 1.48 & 2.07 & 28.55 & 0.54 & 1.01 & 46.80 & 0.48 & 0.85 & 43.31 & 0.37 & 0.58 & 36.77 \\
\hline
\end{tabular}


Accuracy measured by comparing the distance between user constrain $u_{i}$ and its corresponding final position $v_{i}{ }^{\prime}$ as an absolute error $\left(\left|v_{i}^{\prime}-u_{i}\right|\right)$. However, the performance of the surface model always gives better results (mean absolute error) compared to the volumetric model, provides confidence in the surface model to be applied to craniofacial deformation (Table 1).

Other experiments with five fixed constraints (one as handle and four as stationary anchors) are applied to the point cloud of a face point clouds, especially on nasal area. Handle (number 1) is assigned to move toward its original position. Coordinate shifting occurs predominantly in the nasal area, which in this case, is flanked by the four stationary anchors and handles on the tip of the nose (Figure 6 and Figure 7).

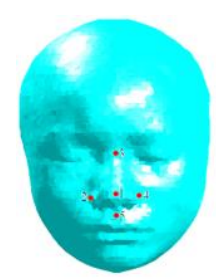

A

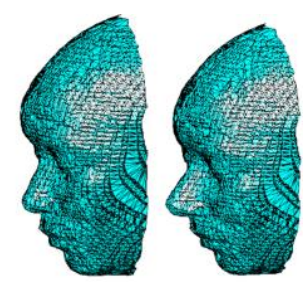

B
Figure 6. User-defined fixed constraints; A. position of constraints (1-5); $B$. The result of augmenting fixed constraints to the deformation process.

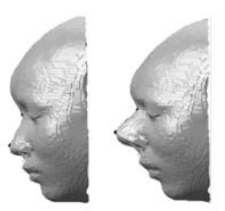

(i)

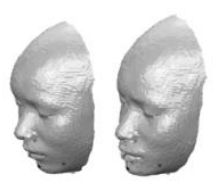

(iv)

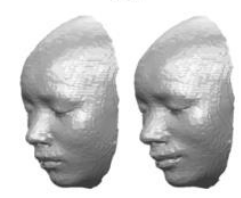

(vii)

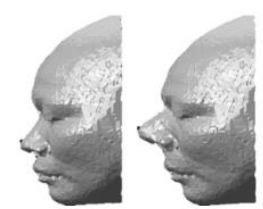

(ii)

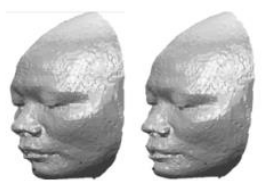

(v)

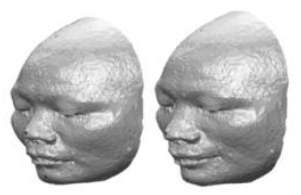

(viii)

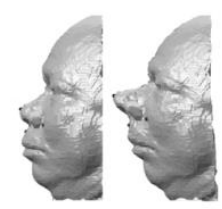

(iii)

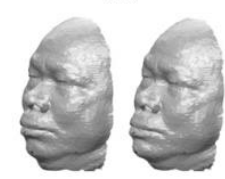

(vi)

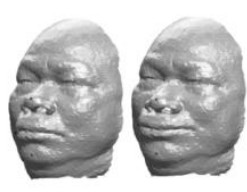

(ix)
Figure 7. Deformation on point clouds of human face using Laplacian surface model; (i, ii, iii) Deformation using five fixed constraints manipulating the shape of nose; (iv, $v$, vi) Chin region manipulation using five fixed constraints; (vii, viii, ix) Seven fixed constraints implemented to create more handle on manipulating cheek.

With the results as in Table 1, the Laplacian surface model is considered better accuracy with $25.9 \%$ to $50.7 \%$ than volumetric Laplacian in fitting face templates to craniofacial reconstruction based on landmark correspondences. Five fixed constraints are applied to facial objects that have been triangulated, focusing on the nose area to see the deformation results. Good results are shown in Figure 6 by using the five constraints with one handle and the other four as the anchors. More experiments are also carried out with seven constraints with two handle configurations and five anchors (Figure 7). Deformation of the specified area is managed by limiting the desired area (RoI) with an anchor and placing the handle at the points within the area where the deformation is desired.

\section{CONCLUSIONS}

Laplacian surface models can be applied well in the deformation of the human face to reconstruct the shape of a person's face based on skull bones. In reconstruction, face templates are fitted to the corresponding face-skull landmarks. The relation with this research is that the fitting method used can be approached with surface deformation using Laplacian surface model. Laplacian surface model is chosen compared to the Laplacian volumetric model because it has better accuracy (with an average $41 \%$ better) considering landmarks fittings in the face reconstruction playing in a narrow range of value. The results obtained from the experiments show that the Laplacian surface model can maintain details in the constellation of human face shape. Reconstruction of the craniofacial based on a priori landmarks knowledge can be conveniently applied with Laplacian surface deformation. Given the role of landmarks in analogy similar to the functions of user fixed constraints, which act as determinants in the deformation.

\section{ACKNOWLEDGMENTS}

Our thanks to Lembaga Pengelola Dana Pendidikan (LPDP)/ Indonesia Endowment Fund for Education, that makes this research possible.

\section{REFERENCES}

[1] Andersson, B. and Valfridsson, M. 2005. Digital 3D Facial Reconstruction Based on Computed Tomography. (2005), 68.

[2] Botsch, M. et al. 2006. Deformation Transfer for DetailPreserving Surface Editing. Vision, modeling and visualization. (2006), 357-364.

[3] de Buhan, M. and Nardoni, C. 2018. A facial reconstruction method based on new mesh deformation techniques. Forensic Sciences Research. 3, 3 (2018), 256-273. DOI:https://doi.org/10.1080/20961790.2018.1469185.

[4] Claes, P. et al. 2010. Computerized craniofacial reconstruction: Conceptual framework and review. Forensic Science International. 201, 1-3 (2010), 138-145. DOI:https://doi.org/10.1016/j.forsciint.2010.03.008.

[5] Dahu, S. and Li, Z. 2012. A fast surface reconstruction algorithm based on Delaunay. Proceedings - 2012 International Conference on Computer Science and Information Processing, CSIP 2012. (2012), 981-984. DOI:https://doi.org/10.1109/CSIP.2012.6309020.

[6] Fang, X. et al. 2015. A Face Matching Method Based on Biometric for Surgical Navigation System. Proceedings 2014 International Conference on Virtual Reality and Visualization, ICVRV 2014. (2015), 412-417. DOI:https://doi.org/10.1109/ICVRV.2014.3.

[7] Fink, M. et al. 2013. Selecting the aspect ratio of a scatter plot based on its delaunay triangulation. IEEE Transactions on Visualization and Computer Graphics. 19, 12 (2013), 2326-2335. DOI:https://doi.org/10.1109/TVCG.2013.187.

[8] Jiang, L. et al. 2010. Craniofacial reconstruction based on MLS deformation. WSEAS Transactions on Computers. 9, 7 (2010), 758-767. 
[9] Li, J. 2011. Nose Surgery Simulation Based on Volumetric Laplacian Deformation. International Joint Conference of IEEE TrustCom (2011), 1482-1487.

[10] Mostafa, S. et al. 2016. An Approach to Effective 3D Reconstruction Based on Point Cloud Merging. 2016 IEEE International WIE Conference on Electrical and Computer Engineering (WIECON-ECE) 19-21 December 2016, AISSMS (2016), 19-21.

[11] Orts-Escolano, S. et al. 2013. Point cloud data filtering and downsampling using growing neural gas. Proceedings of the International Joint Conference on Neural Networks. (2013), 1-8. DOI:https://doi.org/10.1109/IJCNN.2013.6706719.

[12] Romeiro, R. et al. 2014. Forensic facial reconstruction using mesh template deformation with detail transfer over HRBF. Brazilian Symposium of Computer Graphic and Image Processing. (2014), 266-273. DOI:https://doi.org/10.1109/SIBGRAPI.2014.25.

[13] Rosario Campomanes-Álvarez, B. et al. 2015. Modeling Facial Soft Tissue Thickness for Automatic Skull-Face Overlay. IEEE Transactions on Information Forensics and Security. $10, \quad 10 \quad$ (2015), 2057-2070. DOI:https://doi.org/10.1109/TIFS.2015.2441000.

[14] Scheidegger, C. et al. 2005. Triangulating point set surfaces with bounded error. Symposium on Geometry .... (2005), 63. DOI:https://doi.org/10.2312/SGP/SGP05/063-072.

[15] Skalski, A. et al. 2013. CT segmentation based on MRI images in context of prostate radiotherapy planning. IST 2013 - 2013 IEEE International Conference on Imaging Systems and Techniques, Proceedings. (2013). DOI:https://doi.org/10.1109/IST.2013.6729685.

[16] Sorkine, O. 2006. Differential representations for mesh processing. Computer Graphics Forum. 25, 4 (2006), 789807. DOI:https://doi.org/10.1111/j.1467-8659.2006.00999.x.

[17] Sorkine, O. et al. 2004. Laplacian Surface Editing. (2004).

[18] Sun, J. et al. 2018. Laplacian Deformation Algorithm Based on Mesh Model Simplification. 2018 3rd IEEE International Conference on Image, Vision and Computing, ICIVC 2018. 209-213. DOI:https://doi.org/10.1109/ICIVC.2018.8492861.

[19] Ulinuha, M.A. et al. 2017. Outer contour extraction of skull from CT scan images. IOP Conference Series: Materials Science and Engineering. 185, 1 (2017). DOI:https://doi.org/10.1088/1757-899X/185/1/012028. 